\title{
The interplanetary causes of geomagnetic activity during the 7-17 March 2012 interval: a CAWSES II overview
}

\author{
Bruce T. Tsurutani ${ }^{1}{ }^{*}$, Ezequiel Echer ${ }^{2}$, Kazunari Shibata ${ }^{3}$, Olga P. Verkhoglyadova ${ }^{1,4}$, Anthony J. Mannucci ${ }^{1}$, \\ Walter D. Gonzalez ${ }^{2}$, Janet U. Kozyra ${ }^{5}$, and Martin Pätzold ${ }^{6}$ \\ 1 Jet Propulsion Laboratory, California Institute of Technology, Pasadena, CA 91109, USA \\ *Corresponding author: bruce.tsurutani@jpl.nasa.gov \\ 2 The Brazilian Institute for Space Research (INPE), Sao Jose dos Campos, SP 12227-010, Brazil \\ 3 Kwasan Observatory, Kyoto University, 607-8471, Japan \\ 4 Center for Space and Aeronomy Research, University of Alabama, Huntsville, AL 35805, USA \\ 5 University of Michigan, Ann Arbor, MI 48109-2143, USA \\ 6 Department of Planetary Research, The Rhein Institute for the Environment, University of Cologne, 50931 Köln, Germany
}

Received 20 July 2013 / Accepted 11 December 2013

\begin{abstract}
This overview paper presents/discusses the major solar, interplanetary, magnetospheric, and ionospheric features of the CAWSES II interval of study: 7-17 March 2012. Magnetic storms occurred on 7, 9, 12, and 15 March with peak SYM-H intensities of -98 nT, $-148 \mathrm{nT},-75 \mathrm{nT}$ (pressure corrected), and $-79 \mathrm{nT}$, respectively. These are called the S1, S2, S3, and S4 events. Although three of the storm main phases (S1, S3, and S4) were caused by IMF $B_{\text {south }}$ sheath fields and the S2 event was associated with a magnetic cloud (MC), the detailed scenario for all four storms were different. Two interplanetary features with unusually high temperatures and intense and quiet magnetic fields were identified located antisunward of the MCs (S2 and S3). These features are signatures of either coronal loops or coronal sheaths. A high speed stream (HSS) followed the S4 event where the presumably southward IMF $B z$ components of the Alfvén waves extended the storm "recovery phase" by several days. The ICME-associated shocks were particularly intense. The fast forward shock for the S2 event had a magnetosonic Mach number of $\sim 9.4$, the largest in recorded history. All of the shocks associated with the ICMEs created sudden impulses $\left(\mathrm{SI}^{+} \mathrm{s}\right)$ at Earth. The shocks preceding the $\mathrm{S} 2$ and $\mathrm{S} 3$ magnetic storms caused unusually high SI${ }^{+}$intensities of $\sim 60$ and $68 \mathrm{nT}$, respectively. Many further studies on various facets of this active interval are suggested for CAWSES II researchers and other interested parties.
\end{abstract}

Key words. storm - interplanetary coronal mass ejection - shocks - ionosphere (equatorial) - solar wind

\section{Introduction}

The Climate and Weather of the Sun-Earth System (CAWSES) II Workshop, led under the aegis of SCOSTEP, has selected the geomagnetically active period 7-17 March 2012 for an intense group study on all facets of space weather features occurring during this interval. This 11 day period is one of the first major geomagnetically active intervals of the ascending phase of Solar Cycle 24, and therefore is an excellent choice for the group to study.

The purpose of this paper is to identify the major solar and interplanetary causes of geomagnetic activity that occurred as a guide/marker for others who wish to make detailed studies of the solar, interplanetary, magnetospheric, ionospheric, and atmospheric phenomena occurring during the interval. To do this, we will relate solar flares, coronal holes, coronal mass ejections (CMEs), high speed solar wind streams and interplanetary shocks, to effects in the magnetosphere, and ionosphere. It should be noted to the reader that we assume that a CME near the Sun consists of three parts: the bright outer loops, a dark region, and a filament, as defined originally by Illing \& Hundhausen (1986). Because a CME may evolve as it propagates through interplanetary space from the Sun to $1 \mathrm{AU}$, the interplanetary manifestation is called an interplanetary CME or ICME (Tsurutani \& Gonzalez 1997). In this paper, two of the basic structures of a CME are identified and the geoeffectiveness of each are discussed.

\section{Results}

Figure 1 gives an overview of the interplanetary data for the interval of study, showing the ACE solar wind data at $1 \mathrm{AU}$ upstream of the magnetosphere and the geomagnetic responses. From top to bottom the panels are: the solar wind speed, proton density, proton temperature, the magnetic field magnitude, three components of the magnetic field in GSM coordinates, and two geomagnetic activity indices, $A E$ and SYM-H. $A E$ gives a measure of the auroral zone geomagnetic activity, and SYM-H is typically used to identify the occurrence and intensity of magnetic storms and Sudden Impulses ( $\left.\mathrm{SI}^{+} \mathrm{s}\right)$. Decreases in SYM-H (the previously used Dst index has lower time resolution, so is not used here) index indicate enhancements of the ring current energetic $\sim 30-300 \mathrm{keV}$ particles (Dessler \& Parker 1959; Sckopke 1966). When the intensities reach SYM-H $<-50 \mathrm{nT}$, it signifies the occurrence of a magnetic storm (Rostoker \& Falthammar 1967; Gonzalez et al. 1994). This is an arbitrary definition of a storm, but is universally used in practice. There are thus four storms present in this interval using this definition: on March 7, 9, 12, and 15. The peak intensities are $~-98 \mathrm{nT}$, 


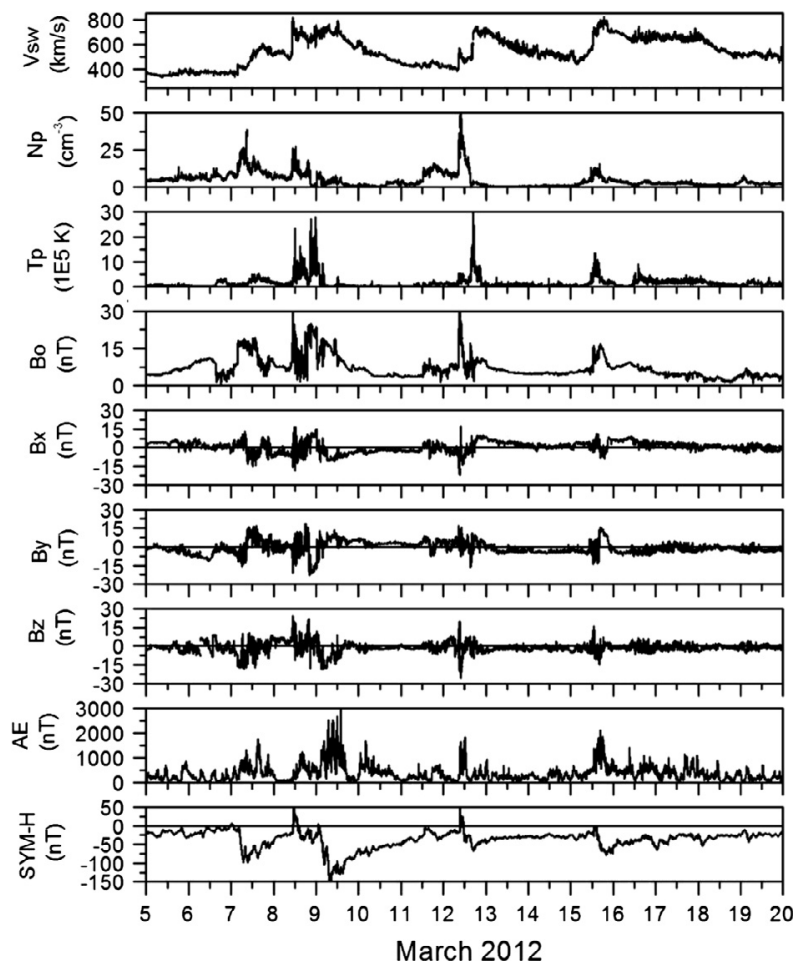

Figure 1. Four magnetic storms (SYM-H $<-50 \mathrm{nT})$ that occurred during the CAWSES interval of study. Peak SYM-H negative values are noted on March 7, 9, 12, and 15. These storm events are called S1 through S4, respectively.

$-148 \mathrm{nT},-67 \mathrm{nT}(-75 \mathrm{nT}$ with solar wind ram pressure corrections), and $-79 \mathrm{nT}$, respectively (pressure corrections were applied to the other three storms, but the effects were small, so they were not included).

For convenience of discussion, these storm events are hereafter referred to as S1 through S4. This designation will be used throughout the paper. All four storms are associated with elevated solar wind speeds (top panel) and enhanced plasma densities and temperatures (second and third panels) and interplanetary magnetic field strengths (fourth panel). The high speeds are associated with the ICMEs and their upstream sheaths and one coronal hole high speed stream. In general, the high plasma densities and temperatures are associated with the interaction of the fast solar wind plasma and magnetic fields (ICMEs) with the slower upstream plasma. Each individual storm will be discussed in some detail below.

\section{Solar causes}

The times, intensities, and solar sites of solar flares associated with the coronal mass ejections (CMEs) are given as columns in Table 1. Solar active region (AR) 1429 was responsible for most of the geomagnetic activity detected at Earth. The largest flare was an X5.4 event and occurred at the beginning of 7 March. With an approximate $\sim 2$ day propagation time for the ICME (we assume the CME was launched at the same time as the flare, the related ICME would have caused the storm on 9 March, or the S2 event. Future modeling will show if this supposition is correct or not. The other solar flares were mostly M-class events. However it is well known that there is not necessarily a one-to-one relationship between the flare intensity and geoeffectiveness of the related ICME (examples are the
Table 1. The flare dates, times, classification and associated AR numbers for the interval 5-15 March 2012.

\begin{tabular}{lccc}
\hline \hline Day & Time max UT & Classification & AR \\
\hline 05 March 2012 & 0409 & X1.1 & 1429 \\
07 March 2012 & 0017 & X5.4 & 1429 \\
07 March 2012 & 0114 & X1.3 & 1430 \\
09 March 2012 & 0353 & M6.3 & 1429 \\
10 March 2012 & 1744 & M8.4 & 1429 \\
13 March 2012 & 1741 & M7.9 & 1429 \\
14 March 2012 & 1521 & M2.8 & 1432 \\
\hline
\end{tabular}

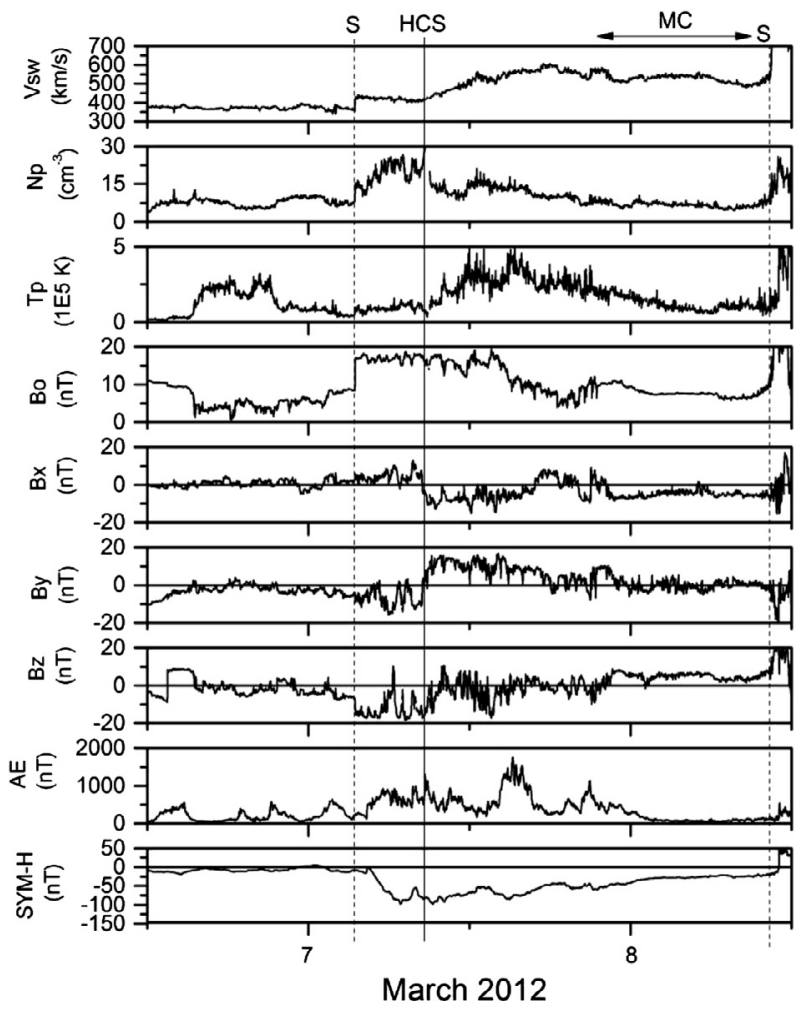

Figure 2. Event S1, the 7 March 2012 storm. The southwardly directed sheath fields caused the magnetic storm main phase.

CAWSES-I ICME events: Tsurutani et al. 2008; Echer et al. 2009).

These major solar flares have been listed for those who wish to study flare spectral changes (Woods et al. 2006) and thus related ionospheric total electron content (TEC) increases due to photoionization (Afraimovich 2000; Tsurutani et al. 2005) and resultant atmospheric heating (Wang et al. 2013). Another topic is modeling of CME release and propagation through interplanetary space to the Earth. It is noted that during times when solar Active Regions are present at the Sun, multiple flaring and multiple CME releases take place. It is known that ICME propagation models have difficulties in predicting accurate ICME arrival times during such conditions (Echer et al. 2009). Thus the CAWSES II interval represents an interesting challenge for ICME propagation models.

\section{Event S1, 7 March}

The first storm on 7 March is shown in detail in Figure 2. The format is the same as in Figure 1. An interplanetary shock at $\sim 0328$ UT is indicated by an " $\mathrm{S}$ " at the top of the panel and 
a vertical dashed line beneath it. The interplanetary heliospheric current sheet (Smith et al. 1978) is indicated by a solid vertical line.

Magnetic clouds (MCs) are identified by smooth, intense magnetic fields, and low plasma densities (e.g., low plasma $\beta$ regions), and a lack of Alfvén waves (Tsurutani et al. 1988; Tsurutani \& Gonzalez 1994; Farrugia et al. 1997). MCs correspond to the dark portion of a CME. In this case, there is a MC event starting at the latter part of 7 March and continuing into 8 March, where the field magnitude is $\sim 8-10 \mathrm{nT}$ and the density $\sim 3-5 \mathrm{~cm}^{-3}$. The MC is indicated in the figure by a horizontal line. In this case, the MC was characterized by IMF $B_{\text {north }}$ components of $\sim 3-5 \mathrm{nT}$, and the result was geomagnetic quiet $(A E<50 \mathrm{nT})$. See Tsurutani \& Gonzalez (1995) and Du et al. (2011) for discussion of a lack of geomagnetic activity during IMF $B_{\text {north }}$ fields.

The magnetic storm main phase starts at $\sim 0200$ UT and reaches a maximum at $\sim 0515 \mathrm{UT}$. The SYM-H peak intensity was $\sim-98 \mathrm{nT}$. The cause of the magnetic storm is clear. Prior to the interplanetary shock, the IMF was southward (IMF $B z<0$ ). The component intensified to $\sim-17 \mathrm{nT}$ due to shock compression of the fields. The intense southward IMF in the sheath behind the shock correlates well with the SYM-H decrease, with a slight delay. Shock intensification of upstream IMF $B_{\text {south }}$ fields has been previously shown to be a mechanism for shock/sheath causes of magnetic storms (Tsurutani et al. 1988). Through magnetic reconnection (Dungey 1961) between the (southwardly directed) sheath fields and the (northwardly directed) magnetopause fields, solar wind energy was transferred to the magnetosphere, and the storm main phase began. Stated another way, the interplanetary dawn-to-dusk motional $V_{\text {sw }} \times B_{\text {south }}$ electric field was exceptionally high during the storm. The IMF $B_{\text {south }}$ component was $\sim 17 \mathrm{nT}$ and the solar wind speed $\sim 420 \mathrm{~km} / \mathrm{s}$, giving an electric field of $\sim 7.7 \mathrm{mV} / \mathrm{m}$. For a discussion of the relationship between interplanetary electric fields and magnetic storms, we refer the reader to Echer et al. (2008).

\section{Event S2, 9 March}

The magnetic storm on 9 March had a different nature and a different interplanetary cause than that for event S1. S2 is shown in Figure 3. The format is the same as in Figure 1. The storm reached a peak intensity of SYM-H $=-148 \mathrm{nT}$ at 0758 UT on 9 March. This is associated with a $\sim-14.5 \mathrm{nT}$ IMF $B z$ component at the beginning of a MC. The MC interval is marked at the top of the panel. The speed of the MC was $\sim 700-740 \mathrm{~km} / \mathrm{s}$. Although the IMF $B_{\text {south }}$ field was comparable to the one in event $\mathrm{S} 1$, the higher speed led to a higher $V_{\text {sw }} \times B_{\text {south }}$ electric field of $\sim 10.4 \mathrm{mV} / \mathrm{m}$, thus a more intense magnetic storm main phase.

The associated interplanetary event contained an unusual feature. There is a high temperature region between $\sim 1130$ UT 8 March and $\sim 0150$ UT 9 March. It has been shaded for emphasis. The high temperatures of $\sim 20 \times 10^{5} \mathrm{~K}$ are unusual this far downstream (toward the Sun) from the shock. It is noted that at the same time the magnetic field reached its highest value for the event ( $\sim 24 \mathrm{nT}$, except for that immediately behind the shock), and there are exceptionally low plasma densities of $\sim 1 \mathrm{~cm}^{-3}$. This occurs upstream of the MC and could be either a coronal loop that has propagated outward from the Sun (Tsurutani et al. 1998) or possibly a more complex "coronal sheath" where coronal plasma has been swept up by the

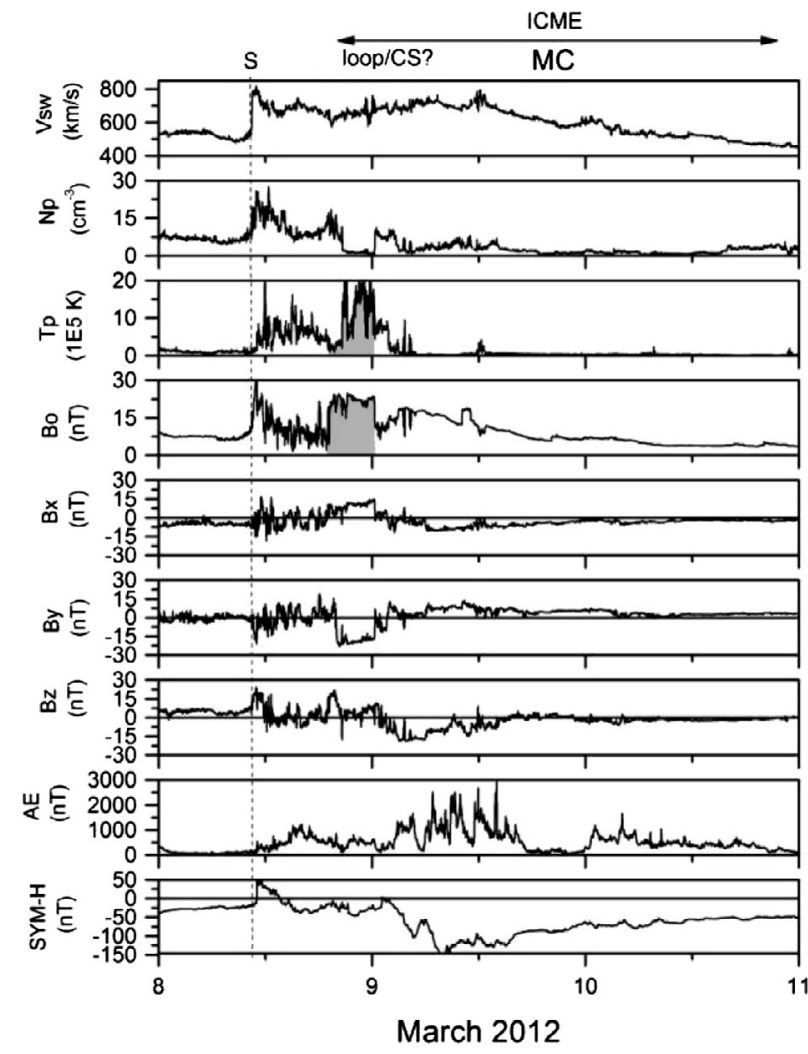

Figure 3. Event S2, 9 March. The storm is caused by the southward field component of a MC. An unusually intense $\mathrm{SI}^{+}$is associated with a strong shock leading the MC. There may be a coronal loop/ coronal sheath detected within the interplanetary (ICME) event (shaded).

outward propagating CME (DeForest et al. 2013). Further analyses should be performed to determine which of these possibilities is most likely.

A strong magnetospheric/ground level feature associated with this event is an unusually intense sudden impulse ( $\mathrm{SI}^{+}$) that occurred at $\sim 1130$ UT 8 March. $\mathrm{A} \mathrm{SI}^{+}$is a sudden positive increase in the horizontal component of the Earth's surface magnetic field induced by shock compression of the magnetosphere (Joselyn \& Tsurutani 1990; Araki et al. 1993, 2009). This event was unusually large, $\sim 60 \mathrm{nT}$ (see bottom panel). The $\mathrm{SI}^{+}$was caused by an intense fast forward shock impinging on the magnetosphere. The solar wind velocity increased from $\sim 525 \mathrm{~km} / \mathrm{s}$ to almost $800 \mathrm{~km} / \mathrm{s}$ across the shock. The density jumped from $\sim 10$ to $\sim 20 \mathrm{~cm}^{-3}$, the temperature from $\sim 6 \times 10^{4} \mathrm{~K}$ to $10 \times 10^{5} \mathrm{~K}$, and the magnetic field from $\sim 9 \mathrm{nT}$ to $30 \mathrm{nT}$. The plasma momentum increases across the shock caused the sudden magnetospheric compression and thus the $\mathrm{SI}^{+}$. This unusually intense shock and its effects will be discussed in more detail later.

\section{Event S3, 12 March}

Figure 4 shows the storm on 12 March 2012, event S3, in high resolution. This event is a "double shock" event, one at $\sim 1228$ UT on 11 March and a second at $\sim 0830$ UT on 12 March. The shock on 11 March created an enhanced plasma density and magnetic field intensity in the downstream sheath. The plasma density increased from $\sim 4$ to $13 \mathrm{~cm}^{-3}$ and the magnetic field increased from $\sim 4$ to $8 \mathrm{nT}$ across the shock. 


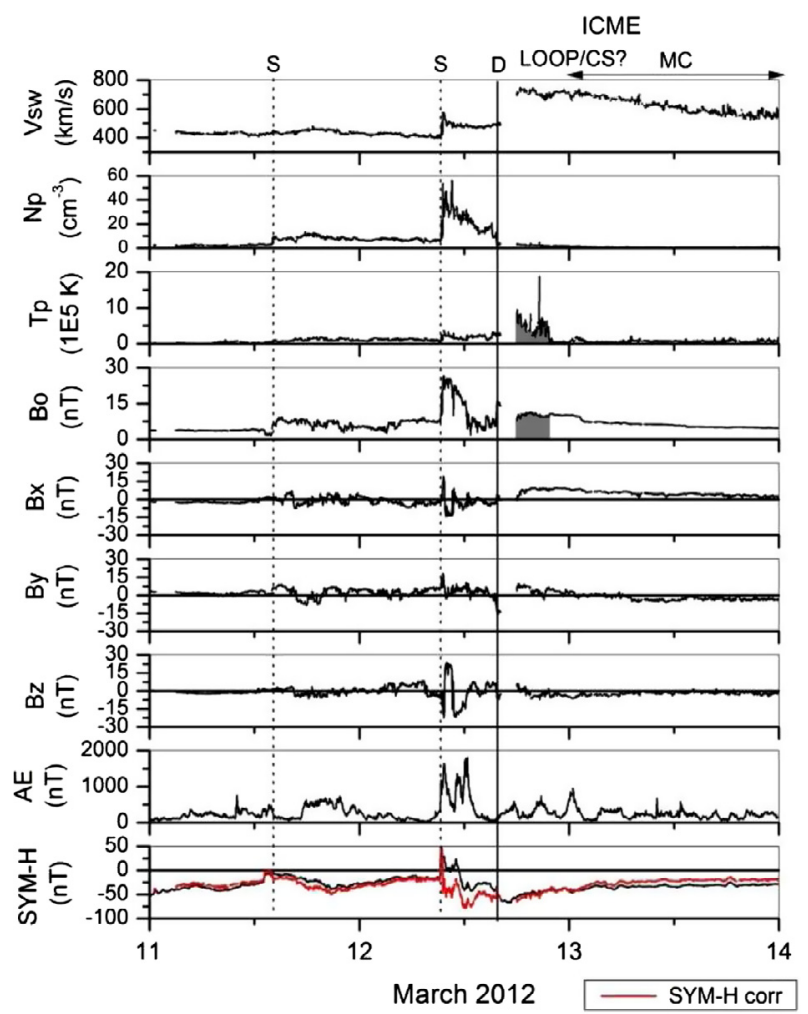

Figure 4. Event S3, 12 March. The S3 storm is caused by southward sheath fields following a second interplanetary shock. A possible loop/coronal sheath and a MC are present in the ICME. Neither of the latter features were geoeffective.

The second shock on 12 March, occurring almost a day later, further enhanced the already large plasma densities and magnetic fields. The density downstream of this second shock was $\sim 48 \mathrm{~cm}^{-3}$ and the magnetic field $\sim 29 \mathrm{nT}$. It is the southward component of this high-intensity magnetic field that is the cause of the S3 magnetic storm. Immediately downstream of the second shock is an intense $B_{\text {north }}$ then $B_{\text {south }}$ variation of the $B z$ fields. The $B_{\text {south }}$ component reached a peak value of $\sim-26 \mathrm{nT}$ and that led to the peak storm main phase (through magnetic reconnection). The peak (solar wind pressure-corrected) SYM-H (shown in red in the bottom panel) was $\sim-75 \mathrm{nT}$. The index was corrected for the solar wind ram pressure, following the Burton et al. (1975) and Gonzalez et al. (1989) formulation. This correction was needed because of the very high solar wind densities occurring in this case. Note that the SYM-H peak without correction (shown in black) would have been only $-66 \mathrm{nT}$, and would have reached its maximum excursion later in time. At the present time, the $B_{\text {north }} / B_{\text {south }}$ field variation is unexplained. Perhaps future stream-stream interaction modeling might shed light on the cause.

The shock on 12 March triggered a "supersubstorm", with $A E>1500 \mathrm{nT}$. Shock triggering of substorms has been well known to occur for some time (Heppner 1955; Kawasaki et al. 1971). More recently Zhou \& Tsurutani (2001) and Tsurutani \& Zhou (2003) have shown that substorms occur within minutes of the shock impingement on the dayside magnetopause and only take place when the magnetosphere has been primed by southward IMFs a few hours prior to impingement (Zhou \& Tsurutani 2001). When priming has not occurred, little or no geomagnetic activity takes place. Such substorm trigger- ing by the many shocks that take place in this interval is a possible study for interested CAWSES II investigators.

What are the two shocks due to? It is assumed that all interplanetary shocks detected to date are "driven" by ICMEs. This assumption has been made by the interplanetary community because no clearly identified "blast wave" shocks have been detected at $1 \mathrm{AU}$ to date. Thus both shocks were most likely driven by ICME "pistons", one of which was not detected by the ACE spacecraft. From past case studies, it is probable that the ICME associated with the first shock was the one that was missed. Further CAWSES II studies should be able to clarify whether this is the case or not.

There are other ICME features that follow later in the event. However because they are not causes of obvious geomagnetic activity, we will only describe them briefly. At $\sim 1500$ UT, there is an unusual discontinuity, indicated by a " $\mathrm{D}$ " and a vertical solid line. The character of the plasma and magnetic field change sunward of the discontinuity. The solar wind speed increases from $\sim 490 \mathrm{~km} / \mathrm{s}$ to $\sim 700 \mathrm{~km} / \mathrm{s}$ and the density decreases from $\sim 10$ to $\sim 3 \mathrm{~cm}^{-3}$. Unfortunately a data gap prevents more detailed analyses of the discontinuity itself.

From $\sim 1537$ UT to 2100 UT, an interval immediately following the above discontinuity, there is a region of high temperature $\sim 3 \times 10^{5} \mathrm{~K}$, low density $\left(\sim 2 \mathrm{~cm}^{-3}\right)$, and high magnetic field $(\sim 7 \mathrm{nT})$. This may be another coronal loop/coronal sheath. The loop/sheath has been shaded for emphasis.

There is a low density, high and smooth magnetic field region following the loop/sheath. This occurs from $\sim 2105$ UT 12 March to $\sim 0000$ UT 14 March. These properties identify this interval as a MC. The $B z$ component within the $\mathrm{MC}$ was $\sim 0 \mathrm{nT}$, so it was not geoeffective.

\section{Event S4, 15 March}

Figure 5 shows the S4 event on 15 March. The storm had a peak intensity of SYM-H $=-79 \mathrm{nT}$ at $\sim 1956 \mathrm{UT}$. Prior to the interplanetary shock which occurred at $\sim 1233$ UT, the IMF $B z$ had a value of $\sim-5 \mathrm{nT}$, which led to a SYM-H of $\sim-28 \mathrm{nT}$. The IMF intensity was $\sim 5 \mathrm{nT}$. The intensity increased to $\sim 15 \mathrm{nT}$ in the postshock region and $B z$ had a range between -5 and $-15 \mathrm{nT}$. It is this latter sheath magnetic field that led to the onset of the storm main phase.

This interplanetary event also contained a MC and a high speed stream (HSS). The MC occurred from 2135 UT 15 March to 1050 UT 16 March. The beginning portion of the MC had southward IMF $B z$ and that also contributed to the main phase of the storm. The HSS began at $\sim 1200$ UT 16 March and lasted until at least $\sim 0200$ UT 18 March. The high-intensity, long-duration, continuous $A E$ activity (HILDCAA; Tsurutani \& Gonzalez 1987) event associated with the HSS event (see the $A E$ panel) kept the SYM-H value at a constant $\sim-25$ to $-30 \mathrm{nT}$ throughout this interval, and led to an extended storm "recovery" phase.

\section{Interplanetary shocks and sudden impulses $\left(\mathrm{SI}^{ \pm} \mathbf{s}\right)$}

Three of the four storms (S1, S3, and S4) were caused by sheath fields behind strong interplanetary fast forward shocks. The shocks led to strong compressions of the upstream interplanetary magnetic fields. The sheath fields had southward components which led to the storm main phases. In at least one case (S3) a prior shock compressed the field ahead of a second shock, leading to unusually intense sheath magnetic fields. 


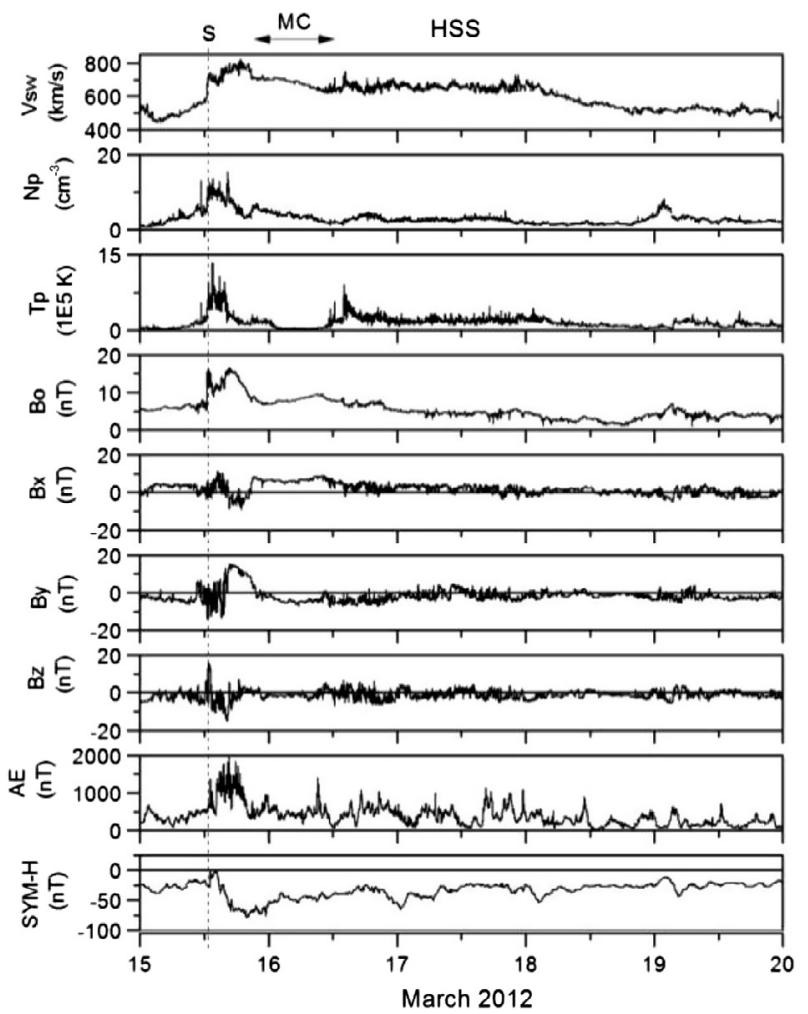

Figure 5. Event S4, 15 March. This storm is caused by $B_{\text {south }}$ sheath fields and the beginning portion of a MC. There is a HSS following the ICME event that causes an extended "recovery" phase to the magnetic storm.

Similar features were noted in the CAWSES I interplanetary events (Tsurutani et al. 2008; Echer et al. 2009). This occurrence of multiple shock compressions occurred before because the CAWSES I organizers selected an interval when a solar active region (AR) was present on the Sun, leading to complex and interactive interplanetary features and thus complex geomagnetic activity. There are frequent flarings at active regions with the consequence of multiple CME releases and thus multiple interplanetary shocks. In this section we give a summary of the analyses of the shock properties.

In all cases, the shock normal directions were determined from the Abraham-Schrauner (1972) mixed-mode method (which uses both plasma and magnetic fields). High time resolution upstream and downstream plasma and field data were used for these analyses. Once the shock normals were determined, the Rankine-Hugoniot conservation relations were applied to obtain the shock speeds. The upstream magnetosonic wave speeds were calculated and the shock Mach numbers obtained. This technique is described by Tsurutani \& Lin (1985) and Echer et al. (2010).

Table 2 gives the results for the five shocks that were discussed previously. The columns are: the event date, the shock time at the WIND spacecraft, the magnetosonic Mach number, the time of the sudden commencement, and the intensity of the $\mathrm{SI}^{+}$.

The shock magnetosonic Mach numbers range from 1.6 to 9.4. The value 9.4 for the S2 event is the largest interplanetary shock that has been detected to date. Interplanetary shock Mach number values typically only vary between $\sim 1$ and 3 (Tsurutani \& Lin 1985; Echer et al. 2010). Thus the magnitude of the event was considerably larger than what is typically observed.
There were clear $\mathrm{SI}^{+}$events for all of the shocks. The $\mathrm{SI}^{+}$ intensities range from $\sim 15$ to $68 \mathrm{nT}$. What is surprising is that the largest event is associated with a Mach 3.4 shock. This is not explained at this time.

Intense interplanetary shocks can have important magnetospheric consequences. For example, a strong interplanetary shock which impacted the magnetosphere on 24 March 1991 created a new radiation belt of $\sim 15 \mathrm{MeV}$ electrons (Vampola \& Korth 1992; Blake et al. 1992; Li et al. 1993) by their imposed $\mathrm{d} B / \mathrm{d} t$ electric fields. It will be interesting to determine if magnetospheric particle acceleration had taken place for these events, and if so, with what intensities.

Intense shocks can also cause the formation of dayside auroras in the auroral zone and at lower latitudes (Craven et al. 1986; Zhou \& Tsurutani 1999; Tsurutani et al. 2001; Zhou et al. 2003). This phenomenon has most certainly occurred at the time of these shocks. The intensity and extent of the particle precipitation needs to be investigated.

Detailed studies of this topic by CAWSES II researchers may provide new and interesting results. Shock compression of preexisting magnetosphere $\sim 10-100 \mathrm{keV}$ energetic electrons and ions may cause plasma instabilities resulting in the growth of electromagnetic electron and ion cyclotron waves (Zhou \& Tsurutani 1999; Tsurutani et al. 2001). Relativistic electrons that gradient drift into this local time sector could parasitically interact with the waves and lead to particle losses to the ionosphere (Clilverd et al. 2012).

Much like the case of interplanetary fast shocks triggering magnetospheric substorms, it has been speculated that "sympathetic flares" that occur at the Sun are an analogous process: solar (photospheric) shocks associated with flaring may trigger primed coronal loops (Tsurutani et al. 2009). It will be interesting to note whether sympathetic flares have occurred during this study interval and if evidence for prior priming can be detected.

The shock triggered supersubstorms in the magnetosphere warrant researchers' attention. Those interested in magnetospheric/magnetotail energy storage and release will have a number of excellent events to study. It has been argued that cases like these may be the best chance for identifying the physics of magnetotail energy storage and release because of the unambiguous timing of the external (interplanetary) trigger (Tsurutani \& Zhou 2003).

\section{Summary and discussion}

The CAWSES II interval had four magnetic storms (events S1-S4): on 7, 9, 12, and 15 March 2012, respectively. The storms could, in general, be associated with solar flares and their related fast CMEs from the solar 1429 AR. However modeling of ICME propagation from the Sun to Earth should be performed before causative relationships can be determined with any certainty. The largest storm on 9 March (SYM$\mathrm{H}=-148 \mathrm{nT}$ ) was associated with a X5.4 flare (the largest flare of the interval) and an ICME that arrived at Earth $\sim 2$ days later. It was the IMF $B_{\text {south }}$ within the related MC portion of the ICME that caused the storm through magnetic reconnection. On the other hand, the magnetic storms of 7, 12, and 15 March were caused/initiated by southwardly directed sheath fields downstream of the ICME shocks.

The CAWSES II interval contains some unusual interplanetary features. Two of the interplanetary events contained regions of unusually high temperatures, low densities, and high 
Table 2. Interplanetary shocks and related sudden impulses ( $\left.\mathrm{SI}^{+} \mathrm{s}\right)$. The columns give (from left to right) the days and times of shock detections at WIND, the magnetosonic Mach number, and the times and intensities of the sudden impulses $\left(\mathrm{SI}^{+} \mathrm{s}\right)$.

\begin{tabular}{lcccc}
\hline \hline Day & Shock time at WIND (UT) & Shock Magnetosonic Mach number & SI $^{+}$time $(\mathrm{UT})$ & $\mathrm{SI}^{+}(\mathrm{nT})$ \\
\hline 07 March 2012 & $\sim 0328$ & 1.8 & $\sim 0420$ & $\sim 1130$ \\
08 March 2012 & $\sim 1026$ & 9.4 & $\sim 1300$ \\
11 March 2012 & $\sim 1228$ & 1.6 & $\sim 0915$ \\
12 March 2012 & $\sim 0830$ & 3.4 & $\sim 1310$ & 15 \\
15 March 2012 & $\sim 1230$ & 2.9 & 68 \\
\hline
\end{tabular}

magnetic field magnitudes (8 and 12 March). In both cases, these regions were spatially located between the sheath and the MC, so they could be signatures of coronal loops or coronal sheaths. These events had similarities and some slight differences with the coronal loop previously reported by Tsurutani et al. (1998). The coronal loop of 10 January 1997 had a magnetic field intensity of $\sim 12 \mathrm{nT}$ with a plasma density of $\sim 7 \mathrm{~cm}^{-3}$. For comparison, the S2 and S3 events here had values of $24 \mathrm{nT}$ and $<1 \mathrm{~cm}^{-3}$ and $\sim 7 \mathrm{nT}$ and $\sim 2 \mathrm{~cm}^{-3}$, respectively. Although the high field intensities noted here are in agreement with the characteristic of a loop, the densities are much lower than previously noted. Thus it is possible that both the loop and the coronal sheath (DeForest et al. 2013) possibilities might not be the explanation here. A third possibility is that these could be MC boundary layers (Galvin et al. 1987) heated by either magnetic reconnection or photoionization from the flare. From the present data one cannot decide which, if any, of these scenarios are correct. More detailed studies using STEREO, interplanetary in situ data and other observations in addition to theoretical modeling are needed to resolve this question.

Kozyra et al. (2013) have speculated that coronal loops/ coronal sheaths at $1 \mathrm{AU}$ are only associated with particularly intense ICME events. This is the case here, in agreement with their conjecture. Although these two events were not geoeffective in a magnetic storm sense (for these specific cases), they may be important for other aspects of the CAWSES II comprehensive study (see the Kozyra et al. results), and thus they have been identified for other people's usage.

There was one high speed stream (HSS) event within the interval. The HSS extended from 16 to 18 March following the $\mathrm{S} 4$ shock/sheath/MC event and had the effect of prolonging the recovery phase of the storm. HSS events are well known to cause geomagnetic activity (Sheeley et al. 1977; Tsurutani et al. 1995; Hajra et al. 2013). It is presumably the southward component of the IMF $B z$ fluctuations within the HSS that is the cause for the continued, high-level auroral zone geomagnetic activity (Tsurutani et al. 2006a, b).

Preconditioning of the magnetosphere for these storms is a topic that could be investigated. In particular Thomsen et al. (2003) have indicated that during northward or near zero IMF $B z$, cold, dense plasma will be accumulated in the Earth's magnetotail. With a subsequent rotation of the IMF to a southward direction, this plasma will be convected into the nightside magnetosphere, forming a particularly intense ring current and enhanced magnetic storm. Kozyra et al. (2013) have noted the significant effect of a cold dense plasma sheet for the 21 January 2005 magnetic storm, but for this event it was not a southward IMF turning, but the high solar wind ram pressure that brought the plasma sheet into the magnetosphere. The S3 event reported here has this northward-southward IMF $B z$ sequence. Three of the magnetic storms (S2, S3, and S4) had exceptionally high ram pressures. It would be interesting to determine if the storm intensities were unusual in any way.
Atmospheric and ionospheric heating by particle precipitation and the resultant disturbance dynamo (Blanc \& Richmond 1980; Proelss 1997) during magnetic storms will cause ionospheric disturbances at auroral and middle latitudes. Storm-time electric fields have strong effects on both the dayside and nightside ionosphere at the equator and at low latitudes (Abdu et al. 1991) if shielding (Tanaka \& Hirao 1973) is suppressed. Penetration of the electric fields lead to depletion of total electron content (TEC) at the magnetic equator during dusk local times (Greenspan et al. 1991) and dayside uplift and substantial enhancement at latitudes away from the equator (Tsurutani et al. 2004; Mannucci et al. 2005). The dayside enhancement has been called the dayside superfountain effect. Strong effects have been noted at dusk as well (Farley et al. 1986; Heelis 2004). Thus the above effects and nightside ionospheric downward convection and density depletion (Tsurutani et al. 2004) are expected during the four storms of this interval. However the extent and intensity of such effects are yet to be investigated by the CAWSES II researchers.

Acknowledgements. Portions of the research were carried out at the Jet Propulsion Laboratory, California Institute of Technology, under contract with NASA.

\section{References}

Abdu, M.A., J.H.A. Sobral, E.R. de Paula, and I.S. Batista, Magnetospheric disturbance effects on the equatorial ionization anomaly (EIA): an overview, J. Atmos. Sol.-Terr. Phys., 53, 757, 1991.

Abraham-Schrauner, B., Determination of magnetohydrodynamic shock normal, J. Geophys. Res., 77, 736, 1972.

Afraimovich, E.L., GPS global detection of the ionospheric response to solar flares, Radio Sci., 35, 1417, 2000.

Araki, T., K. Fumato, T. Iguchi, and T. Kamei, Direct detection of solar wind dynamic pressure effect on ground geomagnetic fields, Geophys. Res. Lett., 20, 775, 1993.

Araki, T., S. Tsunomura, and T. Kikuchi, Local time variation of the amplitude of geomagnetic sudden commencements (SC) and SCassociated polar cap potential, Earth Planets Space, 61, e13, 2009.

Blake, J.B., W.A. Kolasinski, R.W. Fillius, and E.G. Mullen, Injection of electrons and protons with energies of tens of $\mathrm{MeV}$ into L $<3$ on March 24, 1991, Geophys. Res. Lett., 19, 821, 1992.

Blanc, M., and A.D. Richmond, The ionospheric disturbance dynamo, J. Geophys. Res., 85, 1669, 1980.

Burton, R.K., R.L. McPherron, and C.T. Russell, An empirical relationship between interplanetary conditions and Dst, J. Geophys. Res., 80, 4204, 1975.

Clilverd, M.A., D. Danskin, T. Raita, and E.L. Spanswick, Energetic particle injection, acceleration, and loss during the geomagnetic disturbances which upset Galaxy 15, J. Geophys. Res., 117, A12213, DOI: 10.1029/2012JA018175, 2012.

Craven, J.D., C.T. Russell, and R.P. Lepping, Global auroral responses to magnetospheric compressions by shocks in the solar wind: two case studies. In: Sol. Wind-Mag. Coupl., edited by Kamide, Y., and J.A. Slavin, Terra. Sci, Tokyo, 367, 1986. 
DeForest, C.E., T.A. Howard, and D.J. McComas, Tracking coronal features from the low corona to Earth: a quantitative analysis of the 2008 December 12 coronal mass ejection, Astrophys. J., 769, 43, DOI: 10.1088/0004-637X/769/1/43, 2013.

Dessler, A.J., and E.N. Parker, Hydromagnetic theory of magnetic storms, J. Geophys. Res., 64, 2239, 1959.

Du, A.M., B.T. Tsurutani, and W. Sun, Solar wind energy input during prolonged, intense northward interplanetary magnetic fields: a new coupling function, J. Geophys. Res., 116, A12215, DOI: 10.1029/2011JA016718, 2011.

Dungey, J.W., Interplanetary magnetic field and the auroral zones, Phys. Rev. Lett., 6, 47, 1961.

Echer, E., W.D. Gonzalez, B.T. Tsurutani, and A.L.C. Gonzalez, Interplanetary conditions causing intense geomagnetic storms (Dst $\leq-100 \mathrm{nT}$ ) during solar cycle 23 (1996-2006), J. Geophys. Res., 112, A05221, DOI: 10.1029/2007JA012744, 2008.

Echer, E., B.T. Tsurutani, and F.L. Guarnieri, Solar and interplanetary origins of the November 2004 superstorms, Adv. Space Res., 615, DOI: 10.1016/j.asr2009.05.003, 2009.

Echer, E., B.T. Tsurutani, and F.L. Guarnieri, Forward and reverse CIR shocks at 4-5 AU: Ulysses, Adv. Space Res., 45, 798, 2010.

Farley, D.T., E. Bonelli, B.G. Fejer, and M.F. Larsen, The prereversal enhancement of the zonal electric field in the equatorial ionosphere, J. Geophys. Res., 91, 13,723, 1986.

Farrugia, C.J., L.F. Burlaga, and R.P. Lepping, Magnetic clouds and the quiet-storm effect at earth. In: Magnetic Storms, edited by Tsurutani, B.T., et al., Geophys. Mon. Ser., 98, 91:Washington, DC, Amer. Geophys. Un. Press, 1997.

Galvin, A.B., F.M., Ipavich, G. Gloeckler, D. Hovestadt, S.J. Bame, B. Klecker, M. Scholer, and B.T. Tsurutani, Solar wind iron charge states preceding a driver plasma, J. Geophys. Res., 92 (A11), 12,069, 1987.

Gonzalez, W.D., B.T. Tsurutani, A.L.C. Gonzalez, E.J. Smith, F. Tang, and S.-I. Akasofu, Solar wind-magnetosphere coupling during intense magnetic storms (1978-1979), J. Geophys. Res., 94, 8835, 1989.

Gonzalez, W.D., J.A. Joselyn, Y. Kamide, H.W. Kroehl, G. Rostoker, B.T. Tsurutani, and V.M. Vasyliunas, What is a geomagnetic storm?, J. Geophys. Res., 99, 5771, 1994.

Greenspan, M.E., C.E. Rasmussen, W.J. Burke, and M.A. Abdu, Equatorial density depletions observed at $840 \mathrm{~km}$ during the great magnetic storm of March 1989, J. Geophys. Res., 96, 13931, 1991.

Hajra, R., E. Echer, B.T. Tsurutani, and W.D. Gonzalez, Solar cycle dependence of High-Intensity Long-Duration Continuous AE Activity (HILDCAA) events, relativistic electron predictors?, J. Geophys. Res.: Space Phys., 118, 1, DOI: 10.1002/jgra.50530, 2013.

Heelis, R., Electrodynamics in the low and middle latitude ionosphere: a tutorial, J. Atmos. Sol.-Terr. Phys., 66, 825, 2004.

Heppner, J.P., Note on the occurrence of world-wide SSCs during the onset of negative bays at College, Alaska, J. Geophys. Res., 60, 29, 1955

Huang, Y., A.D. Richmond, Y. Deng, P.C. Chamberlin, and L. Qian, et al., Wavelength dependence of solar irradiance enhancement during X-class flares and its influence on the upper atmosphere, to appear, J. Atmos. Sol.-Terr. Phys., 2013.

Illing, R.M.E., and A.J. Hundhausen, J. Geophys. Res., 91, 10951, 1986.

Joselyn, J.A., and B.T. Tsurutani, A note on terminology: geomagnetic sudden impulses (SIs) and storm sudden commencements (SSCs), EOS, 71, 1808, 1990.

Kawasaki, K., S.-I. Akasofu, F. Yasuhara, and C.-I. Meng, Storm sudden commencements and polar magnetic substorms, $J$. Geophys. Res., 76, 6781, 1971.

Kozyra, J.U., W.B.IV Manchester, C.P. Escoubet, S.T. Lepri, M.W. Liemohn, W.D. Gonzalez, M.W. Thomsen, and B.T. Tsurutani, Earth's collision with a solar filament on January 2005: overview, J. Geophys. Res. Space Phys.,

DOI: 10.1002/jgra.50567, 2013.
Li, X.-L., I. Roth, M. Temerin, J.R. Wygant, M.K. Hudson, and J.B. Blake, Simulation of the prompt energization and transport of radiation belt particles during the March 24, 1991 SSC, Geophys. Res. Lett., 20, 2423, 1993.

Mannucci, A.J., B.T. Tsurutani, B.A. Iijima, A. Komjathy, A. Saito, W.D. Gonzalez, F.L. Guarnieri, J.U. Kozyra, and R. Skoug, Dayside global ionospheric response to the major interplanetary events of October 29-30 2003 "halloween storms", Geophys. Res. Lett., 32, L12S02, DOI: 10.1029/2004GL021467, 2005.

Proelss, G.W., et al., Magnetic storm associated perturbations of the upper atmosphere. In: Magnetic Storms, edited by Tsurutani, B.T. 98, Washington, DC: AGU, 227, 1997.

Rostoker, G., and C.G. Falthammar, Relationship between changes in the interplanetary magnetic field and variations in the magnetic field at the Earth's surface, J. Geophys. Res., 72, 5853, 1967.

Sckopke, N., A general relation between energy of trapped particles and disturbed field near Earth, J. Geophys. Res., 71, 3125, 1966.

Sheeley, J.W., Jr., J.W. Harvey, N.R., and W.C. Feldman, A pictoral comparison of interplanetary magnetic field polarity, solar wind speed, and geomagnetic disturbances: 1973-1976, Sol. Phys., 49, 271, 1977.

Smith, E.J., B.T. Tsurutani, and R.L. Rosenberg, Observations of the interplanetary sector structure up to heliographic latitudes of $16^{\circ}$ : Pioneer 11, J. Geophys. Res., 83 (A2), 717, 1978.

Tanaka, T., and K. Hirao, Effects of an electric field on the dynamical behavior of the ionospheres and its application to the storm time disturbances of the F-layer, J. Atmos. Terr. Phys., 35, 1443,1973

Thomsen, M.F., J.E. Borovsky, R.M. Skoug, and C.W. Smith, Delivery of cold, dense plasma sheet material into the near-Earth region. J. Geophys. Res., 108 (A4), 1151,

DOI: 10.1029/2002JA009544, 2003.

Tsurutani, B.T., and R.P. Lin, Acceleraton of $>47 \mathrm{keV}$ ions and $>2$ keV electrons by interplanetary shocks at $1 \mathrm{AU}, J$. Geophys. Res., 90, 1, 1985.

Tsurutani, B.T., and W.D. Gonzalez, The cause of high-intensity long-duration continuous AE activity (HILDCAAs): interplanetary Alfven wave trains, Planet. Spa. Sci., 35, 405, 1987.

Tsurutani, B.T., W.D. Gonzalez, F. Tang, S.-I. Akasofu, and E.J. Smith, Origin of interplanetary southward magnetic fields responsible for major magnetic storms near solar maximum (1978-1979), J. Geophys. Res., 93 (A8), 8519, 1988.

Tsurutani, B.T., and W.D. Gonzalez, The causes of geomagnetic storms during solar maxima, EOS, 75, 49, 1994.

Tsurutani, B.T., and W.D. Gonzalez, The efficiency of viscous interaction between the solar wind and the magnetosphere during intense northward IMF events, Geophys. Res. Lett., 22, 663, DOI: 10.1029/95GL00205, 1995.

Tsurutani, B.T., W.D. Gonzalez, A.L.C. Gonzalez, F. Tang, J.K. Arballo, and M. Okada, Interplanetary origin of geomagnetic activity in the declining phase of the solar cycle, J. Geophys. Res., 100 (A11), 21,717, 1995.

Tsurutani, B.T., and W.D. Gonzalez, The interplanetary causes of magnetic storms: a review. In: Magnetic Storms, edited by Tsurutani, B.T., et al., 98: Washington, DC, Amer. Geophys. Un. Press, 77, 1997.

Tsurutani, B.T., J.K. Arballo, G.S. Lakhina, C.M. Ho, J. Ajello, et al., The January 10, 1997 auroral zone hot spot, horseshoe aurora and first substorm: a CME loop? Geophys. Res. Lett., 25, 3047, 1998

Tsurutani, B.T., X.-Y. Zhou, V.M. Vasyliunas, G. Haerendel, J.K. Arballo, and G.S. Lakhina, Interplanetary shocks, magnetopause boundary layers and dayside auroras: the importance of a very small magnetospheric region, Surv. Geophys., 22, 101, 2001.

Tsurutani, B.T., and X.-Y. Zhou, Interplanetary shock triggering of substorms: WIND and POLAR, Adv. Spa. Res., 31, 1063, 2003.

Tsurutani, B.T., A. Mannucci, B. Iijima, M.A. Abdu, J.H.A. Sobral, et al., Global dayside ionospheric uplift and enhancement associated with interplanetary electric fields, J. Geophys. Res., 109, A08302, DOI: 10.1029/2003JA010342, 2004 
Tsurutani, B.T., D.L. Judge, F.L. Guarnieri, P. Gangopadhyay, A.R. Jones, et al., Geophys. Res. Lett., 32, L03S09,

DOI: 10.1029/2004GL021475, 2005.

Tsurutani, B.T., W.D. Gonzalez, A.L.C. Gonzalez, F.L. Guarnieri, N. Gopalswamy, et al., Corotating solar wind streams and recurrent geomagnetic activity: a review, J. Geophys. Res., 111, A07S01, DOI: 10.1029/2005JA011273, 2006a.

Tsurutani, B.T., R.L. McPherron, W.D. Gonzalez, G. Lu, N. Gopalswamy, and F.L. Guarnieri, Magnetic storms caused by corotating solar wind streams, Recurrent magnetic storms: corotating solar wind streams, 167, Am. Geophys. Un. Press, DOI: 10.1029/167GM03, 2006b.

Tsurutani, B.T., E. Echer, F.L. Guarnieri, and J.U. Kozyra, CAWSES November 7-8, 2004, superstorm: complex solar and interplanetary features in the post-solar maximum phase, Geophys. Res. Lett., 35, L06S05, DOI: 10.1029/2007/GL031473, 2008.

Tsurutani, B.T., K. Shibata, S.-I. Akasofu, and M. Oka, A two-step scenario for both solar flares and magnetospheric substorms: short duration energy storage, Earth Planets Space, 61, 1, 2009.
Vampola, A.K., and A. Korth, Electron drift echoes in the inner magnetosphere, Geophys. Res. Lett., 19, 625, 1992.

Woods, T.N., G. Kopp, and P.C. Chamberlin, Contributions of the solar ultraviolet irradiance to the total solar irradiance during large flares, J. Geophys. Res., 111, A10S14, DOI: 10.1029/2005JA011507, 2006.

Zhou, X., and B.T. Tsurutani, Rapid intensification and propagation of the dayside aurora: large scale interplanetary pressure pulses (fast shocks), Geophys. Res. Lett., 26, 1097, 1999.

Zhou, X., and B.T. Tsurutani, Interplanetary shock triggering of nightside geomagnetic activity: substorms, pseudobreakups and quiescent events, J. Geophys. Res., 106, 18,957, 2001.

Zhou, X.-Y., R.J. Strangeway, P.C. Anderson, D.G. Sibeck, B.T. Tsurutani, G. Haerendel, H.U. Frey, and J.K. Arballo, Shock aurora: FAST and DMSP observations, J. Geophys. Res., 108, 8019, DOI: 10.1029/2002JA009701, 2003.

Cite this article as: Tsurutani B, Echer E, Shibata K, Verkhoglyadova O, Mannucci A, et al.: The interplanetary causes of geomagnetic activity during the 7-17 March 2012 interval: a CAWSES II overview. J. Space Weather Space Clim., 2014,4 , A02. 